\title{
CD200/CD200R PAIRED POTENT INHIBITORY MOLECULES REGULATING IMMUNE AND INFLAMMATORY RESPONSES; PART I: CD200/CD200R STRUCTURE, ACTIVATION, AND FUNCTION
}

\author{
Drahomíra Holmannová ${ }^{1}$, Martina Koláčková ${ }^{1}$, Kateřina Kondělkovál, Pavel Kuneš², Jan Krejsek ${ }^{1}$, Ctirad Andrýs ${ }^{1}$
}

Charles University in Prague, Faculty of Medicine and University Hospital Hradec Králové, Czech Republic: Department of Clinical Immunology and Allergology ${ }^{1}$, Department of Cardiac Surgery ${ }^{2}$

Summary: CD200/CD200R are highly conserved type I paired membrane glycoproteins that belong to the Ig superfamily containing a two immunoglobulin-like domain (V, C). CD200 is broadly distributed in a variety of cell types, whereas CD200R is primarily expressed in myeloid and lymphoid cells. They fulfill multiple functions in regulating inflammation. The interaction between CD200/CD200R results in activation of the intracellular inhibitory pathway with RasGAP recruitment and thus contributes to effector cell inhibition. It was confirmed that the CD200R activation stimulates the differentiation of $\mathrm{T}$ cells to the Treg subset, upregulates indoleamine 2,3-dioxygenase activity, modulates cytokine environment from a Th1 to a Th2 pattern, and facilitates an antiinflammatory IL-10 and TGF- $\beta$ synthesis. CD200/CD200R are required for maintaining self-tolerance. Many studies have demonstrated the importance of CD200 in controlling autoimmunity, inflammation, the development and spread of cancer, hypersensitivity, and spontaneous fetal loss.

Key words: CD200; CD200R; Inhibition mechanisms; Inflammation; IDO

\section{Short introduction of CD200 and CD200R}

The main role of the immune system is to protect the organism against damage by ensuring an adequate response against various pathogens such as viruses, bacteria, fungi, parasites, environmental harm stimuli including allergens, haptens, and also from damage that may result from inappropriate immune system activation. The immune system, therefore, has to comprise mechanisms that maintain homeostasis, including both activatory and inhibitory mechanisms. CD200 and CD200R molecules are involved in the downregulation of myeloid and lymphoid cells. They provide immunomodulatory effects, are able to induce immune tolerance, regulate all differentiation, adhesion, and chemotaxis of various cell populations, mediators and cytokines release. This article aims to summarize the information addressing the regulatory activity of CD200 and CD200R membrane molecules and their putative potential in clinical applications, applications which are, for the moment, tested in vitro or in vivo animal models only.

\section{CD200 and CD200R in detail}

CD200 and CD200R, type I transmembrane-anchored glycoproteins, are structurally similar to immunoglobulins. Their extracellular $\mathrm{N}_{2}$ terminal domain contains both C-type and V-type regions. CD200 and CD200R are identical in their extracellular parts, but they differ in their cytosolic
COOH tails (4). In contrast to CD200R, CD200 have a minimal intracellular region which lacks the capacity to transmit either activatory or inhibitory signals, while the CD200R upon ligation is capable of downregulating the exaggerated activity of the immune system to protect the organism from harm caused by its overactivity. CD200 was first purified and described by Prof. A. N. Barclay and his team in 1981-1982, and it was originally referred to as OX-2. Using mouse monoclonal antibodies, the expression of CD200 was detected in rat lymphoid tissue $(2,3)$. CD200R was depicted later in 2000 by Wright and his team (45). To elucidate expression pattern, gene localization, isoforms and function of CD200 and CD200R, a variety of antibodies has been developed; monoclonal, polyclonal, chimeric, recombinant, together with receptor agonists, full length CD200, or synthesized 9-15 mer peptides defining discrete regions in N-terminal regions of CD200 (14). Finally, transgenic knock-out mice lacking CD200 were generated by using C57BL/6 embryonic stem cells.

\section{1) Expression of CD200/CD200R}

The receptor expression pattern and its ligand show marked differences. CD200 is expressed in a variety of cells both of hematopoietic origin, such as myeloid cells (mast cells, neutrophils, macrophages, dendritic cells), lymphoid cells (T, B cells), and nonhematopoietic origin, such as neurons, cardiomyocytes, endothelial cells, trophoblast 
cells, retinal and optical nerve cells, and keratinocytes (9, 36, 43). Expression of CD200 is enhanced in response to the immune system activation during the inflammatory response. CD200 expression rapidly increases in the presence of TNF $\alpha$, INF $\gamma$ following the activation of TLR-4, scavenger receptor A (SR-A), NALP3 (NACHT-, LRR-, and PYD-containing protein 3), and NOD2 (nucleotide oligomerization domain 2) $(7,8,29)$. Unsurprisingly, apoptotic cells bear a high amount of CD200 at the cell surface. Apoptosis is associated with p53 synthesis, which supports CD200 expression. CD200 gene contains p53 response element sequences that activate p53 mediated gene expression (35). CD200 in apoptotic cells prevents the development of autoimmune diseases. CD200R expression is restricted to lymphoid cells, to T, B cells, NK, and NKT cells, and to myeloid cells. Myeloid cells include dendritic cells, mast cells, eosinophils, basophils, neutrophils, and macrophages, especially subpopulation M2a regulatory macrophages (44). CD200R can be used as a specific marker for M2a macrophages. Differentiation of macrophages to the M2a subset is mediated by IL-4, IL-13, whereas differentiation to M2c macrophages is induced by IL-10 and glucocorticoids (25). The density of CD200R expression varies among distinct leukocyte subsets. The expression of CD200R was identified in mouse leukocytes harvested from the spleen using standard staining and flow cytometric analysis. It was shown that CD200R is highly expressed in CD11 $\mathrm{b}^{+}$cells. It has been well documented that helper $\mathrm{CD}^{+} \mathrm{T}$ cells showed more intensive expression of CD200R compared to $\mathrm{CD}^{+}$ cytotoxic T cells. Helper CD4 ${ }^{+}$effector/memory cells express CD200R with a higher intensity compared to central memory and naive T cells. The expression of CD200R is significantly higher in Th1 T cells compared to Th2 T cells. $\mathrm{B}$ cell CD200R expression is more pronounced in memory cells and plasma blasts. It was discovered that the absence of CD200/CD200R (CD200-/ mice) does not result in any change in the absolute numbers of T, B, NK cells. The production of specific immunoglobulins showed no changes in mice, suggesting that immunoglobulin serum level is CD200R independent (33).

\section{2) CD200 and CD200R gene encoding and isoforms}

Genes encoding CD200 and CD200R are both located on chromosome 3. Precise localization of CD200 encoding gene on chromosome 3 is at position $3 q 12-13$ (26). Finding CD200R encoding gene was more difficult. Vieites and his team mapped the CD200R gene to chromosome 3, region $3 q 13$, using RT-PCR and cDNA cloning. (cDNA of CD200R encodes 325 amino acids, 28 in signal sequences, 215 in extracellular, 21 in transmembrane and 61 in cytoplasmatic domains.) They detected two different coding regions within chromosome 3 with homology to mouse CD200R encoding for isoforms CD200R2/CD200RLa. The mouse CD200 and CD200R genes are located on chromosome 16 (39). It was revealed that there are several related genes in the mouse genome that encode CD200R isoforms termed CD200RL (receptor like - CDR200RLa-e). CD200RL (including hCD200RLa) is characterized by a truncated cytoplasmatic tail with positively charged lysine residues compared to CD200R (1). There is evidence of its functional association with the DNAX-activating protein of $12 \mathrm{kDa}$ (DAP-12) (31, 44). The expression of CD200RLs does not repeat the same pattern as CD200R. CD200RLa is highly expressed in resting mast cells and dendritic cells in the bone marrow. The $\mathrm{Lb}$ form is preferentially located in the activated mast cells. Concerning the fact that there are more isoforms, researchers had been challenging the question of whether CD200 is a ligand for all types of CD200R and CD200RL. Gorczynski's team announced that CD200 has an affinity to any form of receptor CD200R, including isoforms CD200RL (18). Recently it has been established by Deborah Hatherley's team that CD200 is not a ligand for CD200R isoforms (20). They confirmed their claim using highly specific monoclonal antibodies to CD200Fc fusion protein and BaF cells that express CD200R as well as isoforms of CD200RLs. It was demonstrated that CD200 binds only to CD200R. CD200RL binding by CD200 was very weak, almost undetectable. Hatherley pointed out that Gorzynski failed to consider the structural differences of $\mathrm{N}$-terminal tails among CD200R and CD200RLs and their association with DAP-12 (40). Attention of late has focused on detailed mapping of CD200 molecule structure to find sequences that provide optimal interaction leading to effector cell inhibition. Molecular analysis has been elicited showing that CD200 comprises three domains - CDR1, CDR2, CDR3, typical for immunoglobulin molecules. It has also been recently revealed that both molecules CD200/CD200R interact through their extracellular $\mathrm{NH}_{2}$ domains. More accurately, engagement depends on the "GFCC" amino acid sequence. After this discovery, 15-mer peptides from CD200 domains were synthesized to verify the hypothesis that these peptides are able to promote inhibition of the immune system as CD200 does. Surprisingly, some of these short peptides have shown an antagonistic effect on CD200R, and have canceled CD200-induced suppression. Antagonistic properties were observed in peptides from CDR1 and CDR3 regions, whereas peptides from the FR2CDR25 region functioned as agonists-amplified inhibition $(14,15)$. Further studies proved that there is a simultaneous expression of mRNA for two types of CD200, both the full-length and the short, truncated type. The synthesis of CD200 and CD200tr is controlled by SF2/ASF (alternative splicing factor/splicing factor 2). Exons contain specific short sequences affecting their ability to be spliced - ESE (exonic splicing enhancer). These sequences regulate alternative splicing of mRNA through their direct binding to regulatory protein SF2/ASF. CD200tr levels are determined by binding SF2/ASF to ESE. If there is a deficit of ESE, expression of full-length CD200 decreases. Increased expression of SF2/ASF leads to an increase of CD200 synthesis (23). The structural differences between CD200 and CD200tr are that mRNA for CD200tr 
lacks exon-2. This property of mRNA for CD200tr is responsible for alternative splicing of mRNA, and the transcription starts from the second ATG codon and thus the alternative transcript of CD200 lacks the $\mathrm{NH}_{2}$ domain (5). The CD200 truncated form was supposed to retain the same ability to suppress immune reactions as full-length CD200. It was shown that CD200tr is a natural antagonist of CD200. Its ligation to $\mathrm{CD} 200 \mathrm{R}$ results in $\mathrm{TNF} \alpha$ production and $\mathrm{CTL}$ activation. Both CD200tr and CD200 synthesis is promoted by the presence of LPS. Tumors and viruses block the production of CD200tr to protect themselves against immune responses. CD200tr does not bind isoforms of CD200RL (6).

\section{3) CD200 and CD200R functions}

Once the structure of CD200 and CD200R was described, research projects focused on identifying and describing biological functions of CD200 and CD200R. Transgenic knock-out mice lacking CD200 have allowed researchers to reveal the importance of CD200/CD200R interactions which dampen immune system overactivation. Heterozygous CD200 ${ }^{+-}$and homozygous CD200- ${ }^{-}$mice were bred. Homozygous mice showed no CD200 expression in neurons, B cells, or follicular dendritic cells, whereas expression of CD4, CD8, CD220, CD11b molecules remained unaltered. The $\mathrm{CD} 200^{-} /^{-}$mice were found to exhibit an elevated spleen $\mathrm{CD} 11 \mathrm{~b}^{+}$cell population. The activated $\mathrm{CD} 11 \mathrm{~b}^{+}$cells were predominantly located in the red pulp of the spleen causing the spleen's enlargement but were found also in lymph nodes, especially in the mesenteric lymph nodes (45). There is evidence of increased susceptibility to external stimuli that contribute to autoimmune disease development in both $\mathrm{CD} 200^{-/}$animals and in normal mice after blocking CD200R by its antagonists. MOG (myelin oligodendrocyte glycoprotein) administered to such experimental animals induces experimental allergic encephalomyelitis, resembling multiple sclerosis (21). A vigorous inflammatory response was described in facial nerve transection. Lack of CD200 in neurons and microglias resulted in rapid progression of irreversible destruction of the nerve. Pronounced activation was detected at day 2 after transfection. The peak of reactivity was observed at day 4 in contrast to $\mathrm{CD} 200^{+} /^{+}$mice, in which the peak of activation occurs at day 7 after surgery (19). The disruption of the CD200/CD200R receptor interaction is also closely related to the increased susceptibility to collagen-induced arthritis. Wild mice $\left(\mathrm{CD} 200^{+} /^{+}\right)$are resistant to this disease, as well as to the development of autoimmune uveitis (41). Gorczynski and his team clarified the function of CD200/CD200R interaction on the $\mathrm{NLDC}_{145^{+}}$dendritic cell subpopulation, which is associated with better graft survival in mice. This subpopulation shows high expression of the CD200. Blocking of the CD200R by the administration of a CD200R-Fc fusion protein contributes to graft rejection $(16,17)$. It is useful to note that human CD200 and its receptor are highly conserved, sharing identical amino acid sequences with other mammals (rat, mice). The similarity was demonstrated by the administration of human CD200Fc, which displayed the ability to react with mice CD200R and vice versa (44). Recently, viral CD200 homologues (vOXs) were found in viruses, especially in HHV-6 poxviruses and the myxoma virus. It is supposed that vOX downregulates myeloid cell activity during viral infection, thus enabling the escape of viruses $(13,37)$.

\section{CD200/200R signaling cascade}

Immune response must be of sufficient intensity to eliminate exo- and endogenous antigens. To prevent excessive tissue damage, downregulation of the immune system activity is necessary. The immune system suppression is driven by a variety of biologically active substances and receptors. The majority of inhibitory receptors are closely related to the cytoplasmatic sequence ITIM (immunoreceptor tyrosine-based inhibitory motif). CD200R lacks ITIM domains and its cytoplasmatic tail contains three tyrosine residues $(24,46)$. The most membrane distal tyrosine residue is a part of a NpxY domain (27). This domain binds PTB (phosphotyrosine binding domain) sequences on other molecules - Dok1, Dok2, and Shc. Dok's phosphorylation triggers $\mathrm{SH} 2$, which modulates the interaction among Ras/GAP, Csk and SHIP molecules. Dok1 preferentially recruits Crk and CrkL (adaptor protein containing SH2 and SH3 src homology, CT10 sarcoma oncogene cellular homologue domains that activate Ras and Jun kinase), whereas Dok2 is closely related to RasGAP and NcK protein activation (non-catalytic region of tyrosine kinase adaptor protein) (28). To evaluate tyrosine residues and their role in signaling pathway, all three tyrosine residues were mutated to phenylalanine. As a result, the inhibitory function of CD200R was lost. Mutation of tyrosine residues showed the importance of these three tyrosines. Each of them plays an important role in the signal transmission (34). Zhang and his colleagues, in contrast, claimed that only the first and the third tyrosines are needed for signal conducting from CD200R. The second tyrosine residue was shown to have no significance (46). Upon binding of a ligand or agonist antibody, CD200R becomes phosphorylated on its tyrosine residues. This is associated with propagating the signal from the receptor and inducing the inhibitory adaptor protein Dok1 (downstream of tyrosine kinase) and Dok2. Doks are involved in the Ras GTPase (RasGAP) phosphorylation and activation. Dok1, in addition, recruits SHIP (SH2-containing inositol phosphatase). The activation of RasGAP and SHIP leads to inhibition of MAPK (mitogen-activated protein kinase) and NF- $\kappa \mathrm{B}$. Blocking of the MAPK activation results in decreased degranulation of mast cells, decreased synthesis of plethora of cytokines such as TNF $\alpha$, INF $\gamma$, IL-1, IL-17, IL-6, IL-8, IP-10, MIG, and decreased iNOS activity, whereas the synthesis of anti-infammatory IL-10 and TGF- $\beta$ cytokines increases $(22,32)$. The influence of various cytokines on the expression of CD200/CD200R molecules is shown in Fig. 1. 


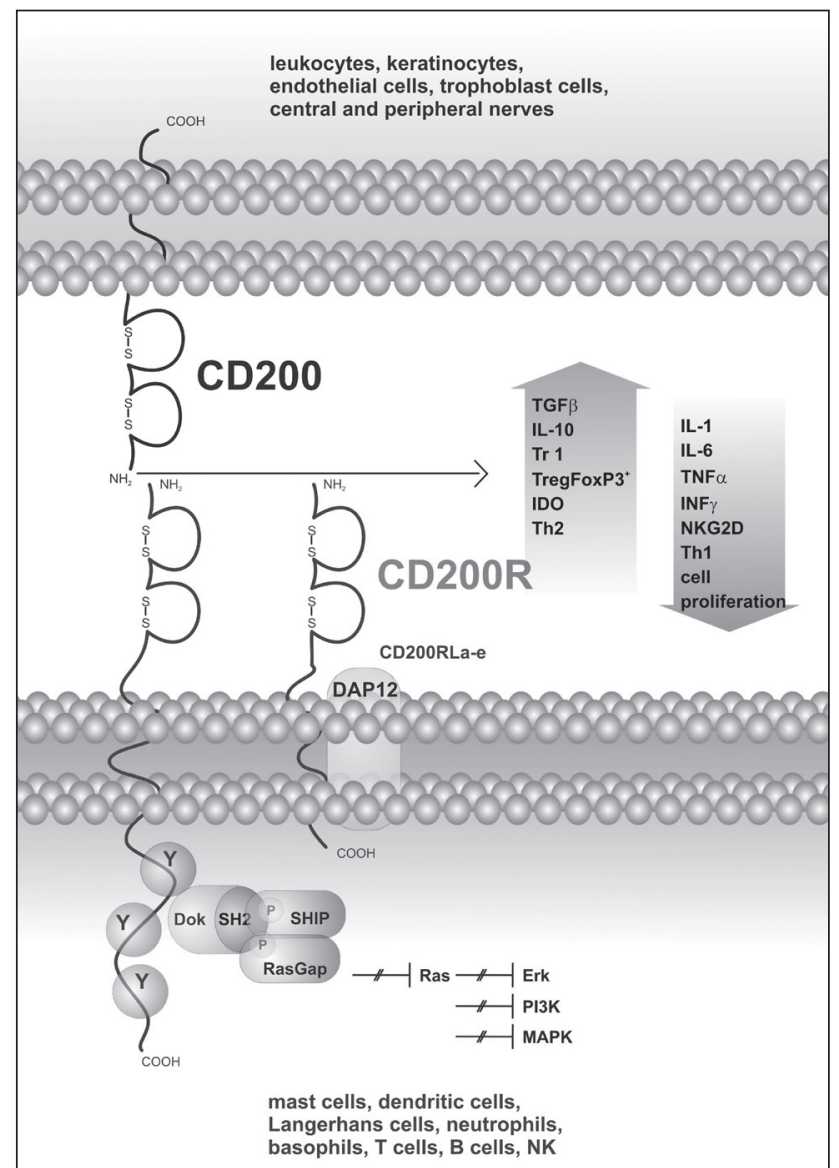

Fig. 1

\section{Inhibition mechanisms}

Direct inhibition of leukocytes is induced by cell-to-cell interaction mediated by CD200/CD200R, which is also responsible for indirect inhibition by polarization of $\mathrm{T}$ cells into either iTreg or Tr1 cells producing IL-10 and TGF- $\beta$. In addition, the shift from Th1 to Th2 cytokine environment or activation of the tryptophan catabolizing enzyme IDO (indoleamine 2, 3-dioxygenase) dependent inhibition pathway is also triggered by CD200/CD200R interaction. IDO is one of the three enzymes metabolizing tryptophan (10). Tryptophan is a substrate for $\mathrm{NAD}^{+}$(nicotinamide adenine dinucleotide) synthesis. Tryptophan is metabolized to kynurenines, which are strong propapoptotic signals. It was determined that IDO suppresses $\mathrm{CD}^{+}$response to ovalbumin in allergic mice. IDO expression, more accurately toxic metabolites of tryptophan, are involved in $\mathrm{CD}^{+} \mathrm{T}$ cell apoptosis. IDO is constitutively expressed by APC cells and trophoblast. However, dendritic cells $\mathrm{CD} 11 \mathrm{c}^{+}$mPDCA- $1^{+} 120 \mathrm{G} 8^{+}$expressed IDO only after activation. The same holds for fibroblasts, astrocytes, eosinophils, and tumor cells. The crucial inducer of IDO expression is the CD200 molecule and its interaction with CD200R. CD200/CD200R interaction directly enables IDO expression or mediates its expression in DC by Treg after CTLA-4 ligation (38). IDO expression is also enabled in the presence of INF $\gamma$, CpG motifs of bacterial DNA. TNF $\alpha$, IL-1, and LPS also strengthen IDO inhibitory potential $(11,42)$. IDO, whose expression is potentiated by CD200/CD200R interaction, suppresses APC cells to prevent autoreactivity and protects the fetus against spontaneous abortion. However, this IDO activity is penalized by an energy deficit as NAD ${ }^{+}$ is decreased in this way (30). As mentioned above, a significantly higher expression of IDO is thought to be associated with inflammation and positively correlates with the severity of inflammation. It follows that a low level of tryptophan is a marker of poor prognosis in autoimmune disease as well as increased IDO expression. As already mentioned in the preceeding text, CD200 supports TGF- $\beta$ synthesis in target cells. This cytokine, as a potent immune inhibitor, blocks the activation of lymphocytes and macrophages, and facilitates differentiation of $\mathrm{T}$ cells to $\mathrm{CD} 4^{+} \mathrm{CD} 25^{+} \mathrm{FoxP} 3^{+}$Tregs, thereby possibly preventing transplanted organs from being rejected or controling and diminishing the autoimmune inflammation (12). The link between CD200/CD200R expression and various diseases is shown in Fig. 2.

\section{Conclusion}

All studies to date confirm that CD200/CD200R interaction promotes inhibitory activities of the immune system. These molecules play an irreplaceable role in maintaining homeostasis, suppressing inflammatory response to both external stimuli (pathogens, allergens, etc.) and internal stimuli (hypoxia, oxygen radicals, tissue damage, etc.). The distribution of CD200 expression is very broad, including immune-privileged tissues, whereas CD200R expression is restricted mainly to myeloid cells that are the first line of cell defense in an organism, the so-called sentinel cells. Downregulation of their activation results in both innate and adaptive immune system inhibition without any alteration of the total cell numbers or immunoglobulin production. Current studies of CD200 and CD200R are aimed at investigating their therapeutic potential for the treatment of diseases associated with increased immune system activity.

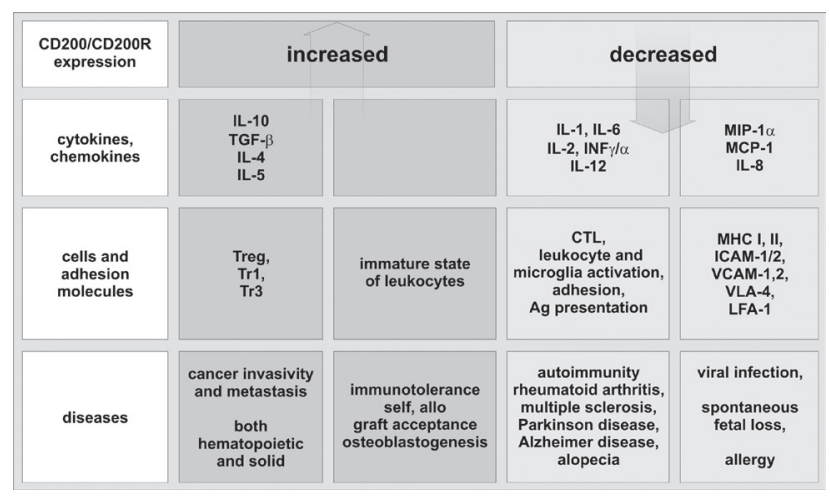

Fig. 2 


\section{Acknowledgements}

This work was supported by Ministry of Education, Czech Republic, project No MSM0021620812 and by a research project No. 262902, granted by Charles University in Prague.

\section{Abbreviations}

APC - Antigen-presenting cell

Csk - C-src tyrosine kinase

CTL - Cytotoxic T-lymphocytes

CTLA-4 - Cytotoxic T-Lymphocyte Antigen 4

DAP-12 - Immunoreceptor tyrosine-based activation motifbearing adapter protein

Dok - Docking protein

ESE - Exonic splicing enhancer

IDO - Indolamine 2,3-dioxygenase

iNOS - Inducible nitric oxide synthese

JNK - Jun N-terminal kinase

LPS - Lipopolysaccharides

MOG - Myelin oligodendrocyte glycoprotein

NK - Natural killer

NKT - Natural killer T (NKT) cells

PTB - Phosphotyrosine binding domain

SF2/ASF - Alternative splicing factor/splicing factor 2

Shc - Phosphotyrosine-binding domain

SHIP - Inositol 5' phosphatase

SH2 - Src homology 2 domain

TLR - Toll like receptor

Treg, Tr1 - Regulatory T cells

vOX2 - Viral CD200 homologue

\section{References}

1. Akkaya M, Barclay AN. Heterogeneity in the CD200R paired receptor family. Immunogenetics 2010; 62(1): 15-22.

2. Barclay AN. Different reticular elements in rat lymphoid tissue identified by localization of Ia, Thy-1 and MRC OX 2 antigens. Immunology. 1981 Dec; 44(4): 727-36.

3. Barclay AN, Ward HA. Purification and chemical characterisation of membrane glycoproteins from rat thymocytes and brain, recognised by monoclonal antibody MRC OX 2. Eur J Biochem. 1982; 129(2): 447-58.

4. Barclay AN, Wright GJ, Brooke G, Brown MH. CD200 and membrane protein interactions in the control of myeloid cells. Trends Immunol 2002; 23(6):285-90.

5. Chen Z, Chen DX, Kai Y, Khatri I, Lamptey B, Gorczynski RM. Identification of an expressed truncated form of CD200, CD200tr, which is a physiologic antagonis of CD200-induced suppression. Transplantation 2008; 86(8): 1116-24.

6. Chen Z, Ma X, Zhang J, Hu J, Gorczynski RM. Alternative splicing of CD200 is regulated by an exonic splicing enhancer and SF2/ASF. Nucleic Acids Res 2010; 38(19): 6684-96.

7. Chen Z, Marseden PA, Gorczynski RM. Role of a distal enhancer in the transcriptional responsiveness of the human CD200 gene to interferon-gama and tumo necrosis factor-alpha. Mol Immunol 2009; 46(10): 1951-63.

8. Chen Z, Marsden PA, Gorczynski RM. Cloning and characterization of the human CD200 promoter region. Mol Immunol 2006 Feb; 43(6): 579-87.

9. Dick AD, Broderick C, Forrester JV, Wright GJ. Distribution of OX2 antigen and OX2 receptor within retina. Invest Ophthalmol Vis Sci 2001; 42(1): 170-6.

10. Fallarino F, Asselin-Paturel C, Vacca C, et al. Murine plasmacytoid dendritic cells initiate the immunosuppressive pathway of tryptophan catabolism in response to CD200 receptor engagement. J Immunol 2004; 173(6): 3748-54.

11. Fallarino F, Orabona C, Vacca C, et al. Ligand and cytokine dependence of the immunosuppressive pathway of tryptophan catabolism in plasmacytoid dendritic cells. Int Immunol 2005; 17(11): 1429-38.

12. Fan H, Wang J, Zhou X, Liu Z, Zheng SG. Induction of antigen-specific immune tolerance by TGF-beta-induced CD4+Foxp3+ regulatory T cells. Int J Clin Exp Med 2009; 2(3): 212-20.
13. Foster-Cuevas M, Wright GJ, Puklavec MJ, Brown MH, Barclay AN. Human herpesvirus $8 \mathrm{~K} 14$ protein mimics CD200 in down-regulating macrophage activation through CD200 receptor. J Virol. 2004 Jul; 78(14): 7667-76.

14. Gorczynski R, Boudakov I, Khatri. Peptides of CD200 modulate LPS-induced TNF-alpha induction and mortality in vivo. J Surg Res 2008; 145(1): 87-96.

15. Gorczynski R, Boudakov I, Khatri I. A comparison of the biological properties of small molecular weight agonists and antagonists of CD200:CD200R interactions. Med Chem 2008; 4(6): 624-31.

16. Gorczynski RM, Cattral MS, Chen Z, et al. An immunoadhesin incorporating the molecule OX-2 is a potent immunosuppressant that prolongs allo- and xenograft survival. J Immunol 1999; 163: 1654-60.

17. Gorczynski RM,Chen Z, Fu XM, Zeng H. Increased expression of the nove molecule OX-2 is involved in prolongation of murine renal allograft survival. Transplantation 1998; 65: 1106-14.

18. Gorczynski R, Chen Z, Kai Y, Lee L, Wong S, Marsden PA. CD200 is a ligand for all members of the CD200R family of immunoregulatory molecules. J Immunol 2004; 172(12): 7744-9.

19. Graeber MB, Lopez-Redondo F, Ikoma E, et al. The microglia/macrophage response in the neonatal rat facial nucleus following axiomy. Brain Res 1998; 813(2): 241-53.

20. Hatherley D, Cherwinski HM, Moshref M, Barclay AN. Recombinant CD200 protein does not bind activating proteins closely related to CD200 receptor. J Immunol 2005; 175(4): 2469-74.

21. Hoek RM, Ruuls SR, Murphy CA, et al. Down-regulation of the macrophage lineage through interaction with OX2 (CD200). Science 2000; 290(5497): 1768-71.

22. Jenmalm MC, Cherwinski H, Bowman EP, Phillips JH, Sedgwick JD. Regulation of myeloid cell function through the CD200 receptor1. J Immunol 2006; 176(1): 191-199.

23. Karni R, de Stanchina E, Lowe SW, Sinha R, Mu D, Krainer AR. The gene encoding the splicing factor SF2/ASF is a proto-oncogene. Nat Struct Mol Biol 2007; 14(3): 185-93.

24. Karra L, Berent-Maoz B, Ben-Zimra M, Levi-Schaffer F. Are we ready to downregulate mast cells? Curr Opin Immunol 2009; 21(6): 708-14

25. Koning N, van Eijk M, Pouwels W, et al. Expression of the inhibitory CD200 receptor is associated with alternative macrophage activation. J Innate Immun 2010; 2(2): 195-200.

26. McCaughan GW, Clark MJ, Hurst J, Grosveld F, Barclay AN. The gene for MRC OX-2 membrane glycoprotein is localized on human chromosome 3 . Immunogenetics. 1987; 25(2): 133-5.

27. Mihrshahi R, Barclay AN, Brown MH. Essential roles for Dok2 and RasGAP in CD200 receptor-mediated regulation of human myeloid cells. J Immunol 2009; 183(8): 4879-86.

28. Mihrshahi R, Brown MH. Downstream of tyrosine kinase 1 and 2 play opposing roles in CD200 receptor signaling. J Immunol 2010; 185(12): 7216-22.

29. Mukhopadhyay S, Plüddemann A, Hoe JC, et al. Immune inhibitory ligand CD200 induction by TLRs and NLRs limits macrophage activation to protect the host from meningococcal septicemia. Cell Host Microbe 2010; 8(3): 236-47.

30. Penberthy WT, Tsunoda I. The importance of NAD in multiple sclerosis. Curr Pharm Des 2009; 15(1): 64-99.

31. Peng Q, Malhotra S, Torchia JA, Kerr WG, Coggeshall KM, Humphrey MB. EM2- and DAP12-dependent activation of PI3K requires DAP10 and is inhibited by SHIP. Sci Signal 2010; 3(122): ra38.

32. Rijkers ES, de Ruiter T, Baridi A, Veninga H, Hoek RM, Meyaard L. The inhibitory CD200R is differentially expressed on human and mouse T and B lymphocytes. Mol Immunol 2008; 45(4): 1126-35.

33. Rijkers ES, de Ruiter T, Baridi A, Veninga H, Hoek RM, Meyaard L. The inhibitory CD200R is differentially expressed on human and mouse $\mathrm{T}$ and $\mathrm{B}$ lymphocytes. Mol Immunol 2008; 45(4): 1126-35.

34. Rijkers ES, de Ruiter T, Verbrugge A, Hoek RM, Meyaard L. CD-200R- mediated inhibition of FceR induced degranulation requires at least two functional tyrosine; Biology of Inhibitory Immune Receptors - Studies on CD200R and LAIR-1, chapter 3, Doctoral study Utrecht University 2007.

35. Rosenblum MD, Olasz E, Woodliff JE, et al. CD200 is a novel p53-target gene involved in apoptosis-associated immune tolerance. Blood 2004; 103(7): 2691-8.

36. Rosenblum MD, Olasz EB, Yancey KB, et al. Expression of CD200 on epithelial cells of the murine hair follicle: a role in tissue-specific immune tolerance? J Invest Dermatol 2004; 123(5): 880-7.

37. Salata C, Curtarello M, Calistri A, Sartori E, et al. vOX2 glycoprotein of human herpesvirus 8 modulates human primary macrophages activity. J Cell Physiol 2009; 219(3): 698-706.

38. Soliman H, Mediavilla-Varela M, Antonia S.Indoleamine 2,3-dioxygenase: is it an immune suppressor? Cancer J 2010; 16(4): 354-9.

39. Vieites JM, de la Torre R, Ortega MA, et al. Characterization of human CD200 glycoprotein receptor gene located on chromosome 3q12-13. Gene 2003; 311:99-104.

40. Voehringer D, Rosen DB, Lanier LL, Locksley RM. CD200 receptor family members represent novel DAP12-associated activating receptors on basophils and mast cells. J Biol Chem 2004; 279(52): 54117-23.

41. Taylor N, McConachie K, Calder C, et al. Enhanced tolerance to autoimmune uveitis in CD200-deficient mice correlates with a pronounced Th2 switch in response to antigen challenge. J Immunol. 2005 Jan 1; 174(1): 143-54. 
42. Trabanelli S, Ocadlikova D, Evangelisti C, Parisi S, Curti A. Induction of regulatory $\mathrm{T}$ cells by dendritic cells through indoleamine 2,3-dioxygenase: A potent mechanism of acquired peripheral tolerance. Curr Med Chem 2011; 18(15): 2234-9.

43. Webb M, Barclay AN. Localisation of the MRC OX-2 glycoprotein on the surfaces of neurones. J Neurochem 1984; 43(4): 1061-7.

44. Wright GJ, Cherwinski H, Foster-Cuevas M, et al. Characterization of the CD200 receptor family in mice and humans and their interactions with CD200. J Immunol 2003; 171(6): 3034-46.
45. Wright GJ, Puklavec MJ, Willis AC, et al. Lymphoid/neuronal cell surface OX2 glycoprotein recognizes a novel receptor on macrophages implicated in the control of their function. Immunity 2000; 13(2): 233-42.

46. Zhang S, Phillips H. Identification of tyrosine residues crucial for CD200R-mediated inhibition of mast cell activation. J Leukoc Biol 2006; 79(2): 363-8.

Received: $16 / 08 / 2011$

Accepted in revised form: 20/03/2012

\section{Corresponding author:}

Prof. RNDr. Jan Krejsek, CSc., University Hospital, Sokolská 581, 50005 Hradec Králové, Czech Republic, Department of Clinical Immunology and Allergology, Czech Republic, e-mail: krejsek@fnhk.cz 